Fetal Diagnosis and Therapy

\title{
Treatment of Primary Fetal Hydrothorax with OK-432 (Picibanil): Outcome in 14 Fetuses and a Review of the Literature
}

\author{
Brooke O'Brien $^{\mathrm{a}}$ Greg Kesby ${ }^{\mathrm{a}}$ Robert Ogle ${ }^{\mathrm{a}}$ Ingrid Rieger ${ }^{\mathrm{b}, \mathrm{c}}$ Jon A. Hyett ${ }^{\mathrm{a}, \mathrm{c}}$ \\ Departments of ${ }^{\mathrm{a}}$ High-Risk Obstetrics and ${ }^{\mathrm{b}}$ Newborn Care, RPA Women and Babies, Royal Prince Alfred Hospital, \\ and ${ }^{C}$ Discipline of Obstetrics, Gynaecology and Neonatology, Faculty of Medicine, University of Sydney, \\ Sydney, N.S.W., Australia
}

\section{Key Words}

Picibanil · OK-432 $\cdot$ Chylothorax $\cdot$ Fetal hydrothorax .

Thoraco-amniotic shunting

\begin{abstract}
Background: Primary fetal hydrothorax (PFHT) is an uncommon condition with an estimated prevalence of 1 in $10,000 / 15,000$ pregnancies. Therapeutic interventions include thoracocentesis, thoraco-amniotic shunting (TAS), and pleurodesis using OK-432. Methods: A review of the literature was performed to identify all cases of PFHT treated with TAS and OK-432. All cases of PFHT referred to the Fetal Maternal Unit at Royal Prince Alfred Hospital between 2002 and 2012 were retrospectively reviewed. In the cohort of fetuses treated with OK-432, the main perinatal outcomes evaluated were termination of pregnancy, live birth, neonatal death, and fetal death in utero. Secondary outcomes included gestational age (GA) at diagnosis, GA at treatment, GA at resolution, birth weight, and GA at birth. The development of the children was screened using the Ages and Stages Questionnaires, Version 3 (ASQ-3, 2009). Results: Primary hydrothorax was diagnosed in 31 fetuses, of which 14 had treatment with OK-432. One pregnancy terminated after treatment with OK-432. Survival was $85 \%$ (11/13): $100 \%$ in fetuses treated with OK-432 without hydrops, and $78 \%$ in
\end{abstract}

those treated with hydrops. This compares well to the cases of TAS in the literature with an average survival of $63 \%: 85 \%$ in fetuses without hydrops and $55 \%$ with hydrops. The mean GA at birth was $36^{+4}$ weeks and mean birth weight 3,007 g. Eight of the 9 children screened with ASQ-3 scored well within the normal range. Conclusion: OK-432 appears to be a valid treatment option in fetuses with PFHT, particularly in those diagnosed at early GAs.

(c) 2015 S. Karger AG, Basel

\section{Introduction}

Primary fetal hydrothorax (PFHT) is uncommon, with an estimated prevalence of 1 in 10,000/15,000 pregnancies [1]. PFHT results from lymphatic fluid accumulation in the pleural space due to anomalies in the lymphatic system. PFHT is described as chylothorax postnatally, once neonatal feeding is established and the lymphatic fluid contains chyle. The diagnosis is made antenatally by a process of exclusion of other underlying immune and non-immune pathologies that can lead to the development of (secondary) pleural fluid collections.

Hydrothoraces vary in volume but as a space-occupying lesion they act to limit lung development and, if large, may cause mediastinal shift (if unilateral) or mediastinal

\section{KARGER 125}

(c) 2015 S. Karger AG, Base

$1015-3837 / 15 / 0374-0259 \$ 39.50 / 0$

E-Mail karger@karger.com

www.karger.com/fdt
Dr. Brooke O’Brien

RPA Women and Babies

Royal Prince Alfred Hospital, Missenden Road

Camperdown, NSW 2050 (Australia)

E-Mail brooke.o’brien@sswahs.nsw.gov.au 
compression (if bilateral), with adverse outcomes including pulmonary hypoplasia, the development of hydrops and polyhydramnios (due to limitations on fetal swallowing), which may result in preterm delivery [2]. Presentation of PFHT varies [3]; some will be noted incidentally at the time of routine obstetric morphology ultrasound assessment, with others detected either incidentally or as a result of investigation of pregnancies considered large for dates, with polyhydramnios, or with reduced fetal movements in the third trimester. Some hydrothoraces will resolve spontaneously in utero [4], whilst others will become progressively more significant [5]. Most commonly, neonates born with a diagnosis of PFHT at or close to term will be effectively treated by simple chest drainage and attention to fluid balance with expectations of resolution within approximately 5-10 days with good long-term outcome. However, in circumstances of long-standing effusions or those complicated by hydrops, the neonatal course may be prolonged and stormy, requiring ventilation, mediumchain fatty acid diets, multiple chest drain insertions and associated higher rates of mortality and morbidity.

Due to the significant morbidity and mortality associated with PFHT, intrauterine therapy is judiciously offered with reference to gestational age (GA), the size of the pleural collections, and the presence of secondary fetal effects, with the aim being one of improving neonatal outcome $[6,7]$. Potential therapeutic interventions include thoracocentesis (either single and multiple procedures), thoraco-amniotic shunting (TAS) [8-13] and, more recently, pleurodesis using OK-432 (Picibanil) [14-22]. The primary aim of all these interventions is to remove fluid from the chest and reduce the adverse effects of the spaceoccupying collection on pulmonary development, cardiovascular function and fetal swallowing. OK-432 has the additional advantage of being able to directly inhibit and possibly cease the fluid production by lymphatic vessels, and additionally effect pleurodesis, which may obviate the need for admission to the neonatal intensive care unit and neonatal drainage. We aim to report our experience using OK-432 as a therapeutic intervention for PFHT and include data on the developmental outcomes of those infants that had a successful neonatal course.

\section{Methods}

To review the literature we evaluated all published studies and case reports of PFHT in peer-reviewed journals over the period 2000-2012. The relevant literature was identified by using PubMed, Embase, and Cochrane databases. In addition, a search was performed of all the reference lists of all identified articles. The key words in the search were fetus, foetus, fetal in combination with hydrothorax, chylothorax, pleural effusion(s), shunting, OK-432, and Picibanil.

The cohort of fetuses treated with OK-432 in this study were identified by retrospective review of consecutive singleton pregnancies complicated by fetal pleural effusion diagnosed at or referred to the Fetal Medicine Unit at Royal Prince Alfred Hospital, Sydney, between 2002 and 2012. Pregnancies complicated by fetal pleural effusion were identified by search of our fetal database (ViewPoint, GE).

PFHT was a working diagnosis defined by the presence of fetal pleural effusions in the absence of other identifiable causes. Secondary pleural effusions were defined as a pleural effusion associated with any fetal malformation, recognised genetic or chromosomal anomaly or other pathology known to result in pleural effusion. Hydrops was defined as the presence of a pleural fluid collection associated with either skin oedema, ascites and/or pericardial effusions. Polyhydramnios was defined as an amniotic fluid index $>25 \mathrm{~cm}$ or maximal single vertical pocket $>8 \mathrm{~cm}$. Effusions were noted to be either unilateral or bilateral and were graded according to size: mild ( $<1 \mathrm{~cm}$ of fluid outlining lungs), moderate $(>1$ $\mathrm{cm}$ of fluid outlining lungs), or severe ( $>1 \mathrm{~cm}$ of fluid outlining lungs with deformation of the mediastinum and/or diaphragm).

Maternal investigations included full blood count, blood group and antibody screen, Kleihauer, serology for parvovirus B19, toxoplasmosis, syphilis, rubella, cytomegalovirus and herpes infections. All fetuses had a morphology ultrasound assessment and echocardiography by a certified maternal fetal medicine specialist. The presence of fetal anaemia was screened for by assessment of middle cerebral artery Doppler peak systolic velocity. Amniocentesis was performed for karyotype and culture/PCR for infective agents (where indicated based on maternal serology). At the same procedure, a (near) complete unilateral fetal thoracocentesis was performed by $20-22 \mathrm{G}$ needle single-puncture aspiration for the purposes of fluid cytology and to assess for recollection prior to embarking on more definitive therapy.

In those fetuses with a working diagnosis of PFHT, both TAS and pleurodesis were discussed with parents as available therapeutic interventions. Intrauterine fetal therapy was offered at a GA of $<35$ weeks in fetuses that were either (1) hydropic where the pleural effusion was suspected as the primary problem or (2) non-hydropic fetuses with recurrent pleural effusion following drainage causing mediastinal deviation or found to be increasing in volume on serial assessment. The therapeutic intervention used in each case was determined by clinical circumstance and the preference of both the treating clinician and the patient who was informed about the potential advantages and risks of each therapy. There was a trend over the period of the study, to move from TAS to OK432 , as clinicians developed more experience with OK-432 as a therapeutic agent. The parents were informed that there were limited reports using OK-432 and gave written consent for the administration of OK-432 as an experimental treatment. Administration involved the transamniotic ultrasound-guided introduction of a 20 $\mathrm{G}$ spinal needle into the fetal thoracic cavity. After drainage of the pleural effusion, 0.1-0.3 KE/kg EFW of OK-432 (Chugai Pharmaceutical Co., Tokyo, Japan) reconstituted in $1.5-2 \mathrm{ml}$ of sterile water was instilled into the pleural space. The procedure was then repeated by separate entry directly into the contralateral hemithorax in the setting of bilateral disease. Ultrasounds were then repeated to monitor the evolution of the effusions at 1-2 weekly in- 
Table 1. Summary of published pleural effusion series reporting intervention

\begin{tabular}{|c|c|c|c|c|c|c|}
\hline & \multicolumn{2}{|c|}{ Number of cases ${ }^{a}$} & $\begin{array}{l}\text { GA intervention, } \\
\text { weeks }\end{array}$ & $\begin{array}{l}\text { GA at delivery, } \\
\text { weeks }\end{array}$ & \multicolumn{2}{|c|}{ Perinatal survival, $\mathrm{n}$} \\
\hline \multicolumn{7}{|l|}{ Shunting } \\
\hline Petersen and Nicolaides [8], 1997 & $41(60)$ & $28(41)$ & 29 & 36 & $19(46)$ & $28(100)$ \\
\hline Picone et al. [9], 2004 & $47(100)$ & - & 31 & 34 & $31(66)$ & - \\
\hline Smith et al. [10], 2005 & $16(76)$ & $5(24)$ & 24 & 32 & $7(44)$ & $3(60)$ \\
\hline Yinon et al. [12], 2012 & $206(74)$ & $72(26)$ & $29^{*}$ & $34^{*}$ & $113(55)$ & $61(85)$ \\
\hline \multicolumn{7}{|l|}{ Pleurodesis OK-432 } \\
\hline Chen et al. [20], 2007 & $3(33)$ & $6(67)$ & 22 & 33 & $0(0)$ & $6(100)$ \\
\hline Nygaard el al. [21], 2007 & $1(14)$ & $6(86)$ & 21 & 38 & $1(100)$ & $6(100)$ \\
\hline
\end{tabular}

Numbers in parentheses are percentages. GA = Median gestational age. ${ }^{*}$ Calculated from studies where data was available.

tervals. The procedure was repeated if there was no evidence of a convincing trend to resolution of the effusion after 2 weeks.

The main outcome measures were resolution of the collection, termination of pregnancy (TOP), live birth (LB), neonatal death (NND), fetal death in utero (FDIU), and childhood developmental outcome. Secondary outcomes included birth weight, GA at diagnosis, GA at treatment, GA at resolution and GA at birth. Childhood developmental outcome was screened using the Ages and Stages Questionnaires, Version 3 (ASQ-3; 2009), a validated developmental assessment tool consisting of a set of 21 questionnaires, each of which has 30 questions adjusted for age groups of children between 1 month and 5.5 years. The questionnaire is completed by parents or primary caregivers and the information sought covers five developmental domains: communication, gross motor, fine motor, problem-solving and personal-social. Administration of the ASQ was performed in consultation with a developmental paediatrician (I.R.). Because the ASQ-3 is a screening tool, completed questionnaires with scores outside the range of normal were scrutinised with a view to further assessment and referral if necessary.

Perinatal outcomes for fetuses with PFHT were analysed for statistical significance using SPSS version 17 (SPSS, Inc., Chicago, Ill., USA), with a p value $<0.05$ considered statistically significant.

This study has ethics approval granted by the Sydney Local Heath District Ethics Committee (Protocol No. X12-0130 and HREC/12/RPAH/221).

\section{Results}

\section{Literature Review}

The prognosis for fetuses with PFHT without hydrops is usually good, with up to $75 \%$ survival and up to a $22 \%$ spontaneous regression rate [3]. However, $57 \%$ of fetuses present with PFHT complicated by hydrops where the prognosis is recognised as being poor, with anticipated survival rates of $24 \%$ without treatment [3]. The presence of severe effusions, bilateral effusions or the development of polyhydramnios are poor prognostic indicators for survival in fetuses that present without hydrops. Thus whilst some authors recommend that intervention should be reserved for hydropic fetuses [23], a justification can be made for therapeutic intervention in cases without hydrops that present with very large or progressive effusions with mediastinal shift and/or demonstrate rapid accumulation of pleural fluid after diagnosis or therapeutic thoracocentesis [11].

Comparison in outcomes between TAS and pleurodesis with OK- 432 are shown in table 1 . There were 278 cases of TAS published in five case series [8-12], with an overall survival rate of $63 \%$ and survival of 55 and $85 \%$ in hydropic and non-hydropic fetuses, respectively. The mean GA at insertion of shunt was 29 weeks, with a GA delivery of 34 weeks. Three case series involving 61 fetuses with PFHT were treated with OK-432 [20-22]. The largest, most recent series of 45 fetuses with PFHTs reported neonatal survival of only $36 \%$ and survival of 15 and $67 \%$ in hydropic and non-hydropic fetuses, respectively. The mean GA of treatment with OK-432 was 24 weeks in the series by Yang et al. [22], with a median GA at delivery of 32 weeks, which was less 
Table 2. Management of 31 fetuses diagnosed with primary fetal hydrothorax

\begin{tabular}{lcllll}
\hline Intervention & Total & TOP & FDIU & NND & LB \\
\hline No treatment & 5 & 0 & 1 & 2 & 2 \\
Pleurocentesis & 6 & 0 & 0 & 1 & 5 \\
Thoracoamniotic shunt & 6 & 0 & 1 & 0 & 5 \\
OK-432 & 14 & 1 & 0 & 2 & 11 \\
\hline & 31 & 1 & 2 & 5 & 23 \\
& & $(3 \%)$ & $(6 \%)$ & $(16 \%)$ & $(74 \%)$ \\
\hline
\end{tabular}

than previously published studies of OK-432, and the current series.

\section{Intervention}

Pleural effusions were diagnosed in 51 fetuses, of which 31 were diagnosed with PFHT. The study cohort consisted of the 14 fetuses treated with OK-432 described in table 2 . There was one TOP at $21+$ weeks after a single dose of OK-432 at $19^{+6}$ weeks in a woman with anxiety disorder due to concern regarding the possible association of the hydrothorax with an undiagnosed syndrome. Subsequent autopsy demonstrated successful pleurodesis and no other fetal anomaly. Of the 13 remaining fetuses treated with OK-432, the number of treatments needed varied, but overall 9/13 (69\%) had a repeat procedure either on one or both sides. The median age for treatment with OK-432 was $24^{+5}$ weeks, and the mean time between first treatment and delivery was 12 weeks.

\section{Pregnancy Outcomes}

Perinatal outcomes of fetuses treated with OK-432 are shown in table 3. Of 13 fetuses treated with OK-432, 11 were born at a GA ranging from 31 to 41 weeks, with mean GA $36^{+4}$ weeks. Resolution of hydrothorax occurred in utero in 8 of 13 infants. The mean birth weight was $3,007 \mathrm{~g}$ (range 1,650-3,795). The mean age at diagnosis of fetal hydrothorax was $23^{+1}$ weeks (range 18-31). Fetuses with hydrops were more likely to be diagnosed and delivered at an earlier gestation than those without, although this trend did not reach statistical significance $(\mathrm{p}=0.22)$.

There were 2 (15\%) NNDs in fetuses that did not have resolution in utero. One infant (case 8) was diagnosed with bilateral hydrothoraces and hydrops at $24^{+1}$ weeks, and had bilateral treatment with OK- 432 at $25^{+1}$ and $27^{+1}$ weeks. From then the pregnancy was seen at the original referral hospital and had persistent small pleural hydro- thoraces with signs of ascites and polyhydramnios seen on ultrasound, and had an amnioreduction performed at $30^{+4}$ weeks. The mother developed preterm labour at $31^{+6}$ weeks, and given her history of more than one previous caesarean delivery, a repeat emergency caesarean was performed. The infant died at $12 \mathrm{~h}$ of life from pulmonary hyperplasia and fetal hydrops, with the cause of death confirmed on autopsy. The second NND occurred in an infant (case 9) with unilateral hydrothorax with hydrops treated at $20^{+5}$ weeks and with subsequent resolution of the hydrothorax documented at 24 weeks' gestation. The infant was found to have recurrence of pleural fluid collections at 31 weeks at the original referral hospital, followed by spontaneous rupture of the membranes at 32 weeks. The infant was delivered electively by caesarean at $32^{+4}$ weeks after administration of antenatal steroids, and had bilateral chest tube insertion but died from pulmonary sepsis in the late neonatal period. Autopsy found bilateral pulmonary consolidation but did not demonstrate any evidence of pulmonary hypoplasia. Both infants had a birth weight $>95$ th percentile, consistent with persistent hydrops.

One child (case 4) was found to have mild left-sided ventriculomegaly and an area of mixed echogenicity in left anterior cerebral hemisphere on ultrasound 3 weeks following the initial treatment with OK-432. MRI at $28^{+4}$ and $32^{+3}$ weeks confirmed a $10 \times 9 \times 10 \mathrm{~mm}$ focal rounded area of increased T2 signal intensity in the deep white matter of the left frontal lobe at the superolateral angle of the frontal horn of the lateral ventricle associated with very mild enlargement of left lateral ventricle, with no other brain abnormality being evident.

\section{Long-Term Outcome}

Nine of the 11 surviving children's parents completed the ASQ-3 developmental screened at ages shown in table 3. Two children were unable to be screened due to their age. However, both are attending school and are performing at an expected level with no concerns expressed by their parents. Eight of the 9 screened children scored well within the normal range. The remaining child (case 12), screened at the age of 60 months, was already known to have both an expressive and receptive language disorder and was receiving intervention.

The child (case 4) that had antenatal ultrasound and MRI changes on CNS examination was followed up within the neonatal department after delivery. An MRI at 1 month of age showed a well-defined cystic cavity measuring $10 \times 5 \times 10 \mathrm{~mm}$; however, there was no evidence of brain abnormality. It was the opinion of the treating neo- 
Table 3. Outcomes and characteristics of cases of fetal hydrothorax treated with OK-432

\begin{tabular}{|c|c|c|c|c|c|c|c|}
\hline Case & Diagnosis & $\begin{array}{l}\text { GA } \\
\text { diagnosis }\end{array}$ & $\begin{array}{l}\text { GA } \\
\text { OK432 }\end{array}$ & $\begin{array}{l}\text { GA } \\
\text { birth }\end{array}$ & $\begin{array}{l}\text { Birth } \\
\text { weight, g }\end{array}$ & Outcome & ASQ, age \\
\hline 1 & Left hydrothorax & $31+4$ & $31+6$ & $37+5$ & 3,285 & LB & Normal, 6 months \\
\hline 2 & Bilateral hydrothorax & $19+5$ & $21+5$ & $39+5$ & 3,365 & LB & Normal, 12 months \\
\hline 3 & Bilateral hydrothorax & $30+0$ & $31+0$ & $32+1$ & 1,650 & LB & Normal, 60 months \\
\hline 4 & Bilateral hydrothorax & $23+0$ & $25+1$ & $41+2$ & 3,468 & LB & Normal, 12 months \\
\hline 5 & Bilateral hydrothorax with hydrops & $19+3$ & $21+2$ & $38+0$ & 3,200 & LB & $\mathrm{NA}^{\mathrm{a}}, 10$ years \\
\hline 6 & Right hydrothorax with hydrops & $19+2$ & $19+5$ & $36+5$ & 2,802 & LB & Normal, 18 months \\
\hline 7 & Left hydrothorax with hydrops & $18+0$ & $19+1$ & $41+1$ & 3,500 & LB & Normal, 48 months \\
\hline 8 & Bilateral hydrothorax with hydrops & $24+1$ & $25+1$ & $31+6$ & 2,795 & NND & - \\
\hline 9 & Left hydrothorax with hydrops & $18+5$ & $20+5$ & $32+4$ & 2,555 & NND & - \\
\hline \multicolumn{2}{|c|}{ With hydrops } & $22+0$ & $23+5$ & $36+1$ & 3,035 & $75 \%$ survival & \\
\hline \multicolumn{2}{|c|}{ All cases } & $23+1$ & $24+5$ & $36+4$ & 3,007 & $85 \%$ survival & \\
\hline
\end{tabular}

${ }^{a}$ Not applicable, above the age for screening with ASQ-3.

${ }^{\mathrm{b}}$ Known to have an expressive and receptive language disorder and verbal dyspraxia.

natologist that this most likely represented a frontal horn cyst [24], which are present in up to $1 \%$ of fetuses $<32$ weeks' gestation and are not associated with any increased risk for adverse neurodevelopmental outcome. ASQ-3 screening in this infant was normal for age, and assessment by the developmental paediatrician (I.R.) at 12 months of age using Bayley-III Scales of Infant Development found normal cognition, language and motor skills and there were no other developmental concerns.

\section{Discussion}

\section{Summary of Findings and Comparison of Interventions}

This cohort provides further evidence for the potential value of OK-432 as a therapeutic agent in the management of primary hydrothoraces. $85 \%$ of infants survived after treatment with OK-432; $100 \%$ of those that were not hydropic and $78 \%$ of those that were hydropic at the time of intervention. GA at diagnosis and treatment with OK432 in this series was $23^{+1}$ and $24^{+5}$ weeks, respectively, which is lower than the reported mean of $25^{+2}$ and $27^{+6}$ weeks for TAS [12]. Earlier onset of primary hydrothorax is known to be associated with a poorer prognosis [3], yet despite this, overall GA at delivery and survival in this cohort was similar to that described in those series where shunts were introduced at later gestations. The less invasive nature of OK-432 may have lowered the author's threshold for intervention, which would benefit the fetus in terms of optimising fetal lung growth and expansion.

The mean GA at birth of OK-432-treated fetuses in this study was $36^{+4}$ weeks, compared to 34 weeks with shunting in the literature. There were 5 infants (38\%) treated with OK-432 that delivered preterm; all premature deliveries occurred in the fetuses where resolution did not occur. Two of the 5 fetuses delivered preterm did not survive; 1 fetus (case 8) developed spontaneous preterm labour at 31 weeks, and a second delivered at 32 weeks following spontaneous rupture of the membranes (case 9). The 3 fetuses that survived were delivered electively at 32-33 weeks for persistent pleural effusions causing fetal compromise, and required admission to the neonatal nursery for drainage of persistent hydrothorax.

The most common complications that occur with TAS are preterm rupture of the membranes (15\%), chorioamnionitis (8.5\%), and preterm delivery (71\%) [9]. In this series there were no cases in which treatment with OK-432 resulted in iatrogenic preterm rupture of the membranes or preterm labour within $48 \mathrm{~h}$ of treatment, 
or in chorioamnionitis. This is an important finding as premature delivery is the leading cause of perinatal morbidity and mortality in these fetuses. Between 32 and 37 weeks, delivery of fetuses with primary hydrothorax increases the risk of death by a factor of $1.3(1.1-1.6)$ for each week of prematurity [3]. The risk of death is 3.6fold higher (1.3-10.3) for an infant born any time before 35 weeks relative to an infant born after 35 weeks. OK432 administration is technically easier to perform than shunt placement - as it can be delivered via a standard $20 \mathrm{G}$ spinal needle rather than the larger 3-mm trocar and cannula used for shunting, making it less invasive and particularly easier to perform at earlier gestations and in hydropic fetuses with significant skin oedema. The injection of OK-432 is likely to have a procedurerelated risk similar to thoracocentesis and amniocentesis, with risk of premature rupture of the membranes of $1-1.6 \%$ and fetal loss of $0.5-1 \%$ [6]. The procedure-related risk of fetal loss with shunting is $5-10 \%$ [6], and up to $30 \%$ of fetuses that have TAS require more than one procedure [12].

Eight fetuses (62\%) in this study had resolution of hydrothorax and/or hydrops in utero following administration of OK-432, which compares well to TAS where a $47.5 \%$ resolution rate is reported [12]. To achieve resolution, $69 \%$ of fetuses in this series required repeat treatment with OK-432. It is possible that the success of treatment in the setting of repeat procedures was in part due to the associated repeated aspirations. However, we believe that the successful outcome achieved after repeated treatment with OK-432 was primarily due to achievement of pleurodesis rather than by virtue of serial thoracocentesis. By achieving pleurodesis and cessation of fluid production, there is the possibility of avoiding postnatal chest tubes and the neonatal nursery altogether. None of the 8 fetuses which achieved resolution in utero in this study required admission to the neonatal nursery for chest drainage.

\section{Safety of OK-432 and Neurodevelopmental Issues}

OK-432 has not been shown to have any serious or permanent sequelae in the paediatric population in which it has been very widely used for treatment of head and neck lymphatic malformations [25]. However, the absolute safety of OK-432 in animal or human fetuses has not been established. There is evidence that exposure of the preterm fetus to infection and inflammation can lead to the development of neural injury and cerebral palsy, based primarily on evidence from exposure to Gramnegative bacteria lipopolysaccharide [26]. OK-432 is derived from a low-virulence $\mathrm{Su}$ strain of type 3 group A
Streptococcus pyogenes (a Gram-positive bacterium) treated with benzylpenicillin (penicillin $G$ ) and heat to eliminate its toxin-producing capability and ability to cause infection. OK-432 acts as an immunostimulant $[27,28]$, promoting a localised inflammatory response by activation of neutrophils, macrophages, lymphocytes and natural killer cells, and induction of multiple cytokines, including IL-1, IL-2, IL-6, TNF and IFN, which causes pleural inflammation and fibrosis resulting in pleurodesis. In a study of 45 pregnant Japanese white rabbits, OK-432 or saline was administered either subcutaneously behind the back of the fetal neck or into the amniotic cavity [29]. No statistical difference in fetal Creactive protein was found between the OK-432 and saline groups after $48 \mathrm{~h}$, indicating that there was no systemic inflammatory response.

In two small studies of 10 and 13 sheep fetuses [30,31], intrathoracic administration of OK-432 under general anaesthesia was found to be associated with very early transient suppression of fetal EEG activity, breathing and body movements without induction of hypotension or hypoxia. The significance of the early transient suppression effect is unknown in humans or animals, and the dose, duration or effect of general anaesthesia was not explored. There was no medium-term effect on brain activity or behaviour in all but 1 sheep fetus that developed seizures $18 \mathrm{~h}$ after administration of OK-432, and died on day 6. On postmortem histological examination of the brain, a large infarct was observed on both sides of the hippocampi. No other histological changes were found in the surviving fetuses postmortem. It is worth noting that several other invasive procedures where performed on these fetuses during hysterotomy including vascular catheters in the femoral artery, brachial artery, and carotid artery, which would all carry a significant risk of embolic events. In this cohort, there was 1 fetus (case 4) identified to have a cerebral lesion at $28^{+1}$ weeks; 3 weeks after OK$432 \mathrm{administration}$. This lesion most likely represented a frontal horn cyst, but it is difficult to completely exclude an adverse event related to administration of OK- 432. This is certainly not a reason alone to abandon treatment with OK-432, but supports the establishment of an international register for use of OK-432 in fetuses with PFHT to monitor treatment success, failure and adverse events.

\section{Limitations of This Study}

The main limitation of this study is that it involves a small number of cases treated with OK-432, and there was the potential for selection and treatment bias. The success of treatment with OK-432 in the literature is likely to be 
inflated due to publication bias, as evidence by the $100 \%$ survival in the two earlier papers compared to the $36 \%$ survival in the larger case series. Better estimates of efficacy of OK-432 may be possible with international, multicentre collaboration and registration of treatments with OK-432. Although this is the first study to attempt to quantify the longer-term outcome in fetuses treated with OK-432, the information on the long-term outcome in this study is limited. Interpretation of neurodevelopment outcome in this study through parental questionnaires has several limitations. Furthermore, 4 of the 9 infants screened were 6-12 months of age at the time of screening, where it can be difficult to determine the presence of developmental delay.

\section{Limitations of Treatment with $\mathrm{OK}-432$}

A potential limitation of therapy with OK- 432 is that some cases of primary hydrothorax appear to be genetic in origin. Familial occurrence in an autosomal recessive inheritance pattern has been observed in four families where chylothorax has affected 2 or more siblings [20, 32]. In our cohort, 1 fetus (case 9) that did not have resolution with OK-432 and had NND had 2 previous siblings affected by PFHT with hydrops; one pregnancy resulting in FDIU at 23 weeks and another resulted in a LB at 32 weeks after in utero treatment with TAS and postnatal chest tube. Additionally, 2 of the 6 fetuses treated with TAS in the group of 51 fetuses with PFHT were offspring from the same mother. Four possible genes involved in thoracic duct or lymphatic vessel development VEGFR3, FOXC2, ITGA9 and ITGB - have been implicated in the pathogenesis of chylothorax [33,34], with up to $30 \%$ fetuses with PFHT having mutations in these four loci [22]. The presence of a mutant allele was associated with a poorer response to OK-432 pleurodesis and a worse prognosis. In fetuses where there is a family history, TAS may remain the gold standard for treatment.

\section{Conclusion}

Fetal pleural effusion is an uncommon anomaly which can be associated with aneuploidy and a range of other structural malformations or genetic syndromes. Intrauterine therapy for an apparently primary hydrothorax will be influenced by the expected impact on fetal wellbeing and GA at presentation. While OK-432 remains an experimental treatment as its safety profile remains to be clarified, this study presents more encouraging human data that OK-432 appears to be a valid treatment option in the setting of PFHT with or without hydrops.

\section{References}

1 Longaker MT, Laberge JM, Dansereau J, Langer JC, Crombleholme TM, Callen PW, Golbus MS, Harrison MR: Primary fetal hydrothorax: natural history and management. J Pediatr Surg 1989;24:573-576.

$\checkmark 2$ Weber AM, Philipson EH: Fetal pleural effusion: a review and meta-analysis for prognostic indicators. Obstet Gynecol 1992;79:281289.

-3 Aubard Y, Derouineau I, Aubard V, Chalifour V, Preux PM: Primary fetal hydrothorax: a literature review and proposed antenatal clinical strategy. Fetal Diagn Ther 1998;13:325333.

4 Rustico MA, Lanna M, Coviello D, Smoleniec J, Nicolini U: Fetal pleural effusion. Prenat Diagn 2007;24:793-799.

5 Klam S, Bigras JL, Hudon L: Predicting Outcome in primary fetal hydrothorax. Fetal Diagn Ther 2005;20:366-370.

6 Deurloo KL, Devlieger R, Lopriore E, Klumper FJ, Oepkes D: Isolated fetal hydrothorax with hydrops: a systematic review of prenatal treatment options. Prenat Diagn 2007;27: 893-899.
7 Vaughan J, Fisk NM, Rodeck CH: Fetal pleural effusion; in Harman CR (ed): Invasive Fetal Testing and Treatment. Boston, Blackwell Scientific, 1995, pp 219-239.

8 Pettersen HN, Nicolaides KH: Pleural effusions; in Fisk NM, Moise KJ (eds): Pleural Effusions. Cambridge University Press, Cambridge, 1997, pp 261-272.

-9 Picone O, Benachi A, Mandelbrot L, Ruano R, Dumez Y, Dommergues M: Thoracoamniotic shunting for fetal pleural effusions with hydrops. Am J Obstet Gynecol 2004;191:20472050.

10 Smith RP, Illanes S, Denbow ML, Soothill PW: Outcome of fetal pleural effusions treated by thoracoamniotic shunting. Ultrasound Obstet Gynecol 2005;26:63-66.

11 Pellegrinelli JM, Kohler A, Kohler M, Weingertner AS, Favre R: Prenatal management and thoracoamniotic shunting in primary fetal pleural effusions: a single-centre experience. Prenat Diagn 2012;32:467-471.

12 Yinon Y, Grisaru-Granovsky S, Chaddha V, Windrim R, Seaward PG, Kelly EN, Beresovska O, Ryan G: Perinatal outcome following fetal chest shunt insertion for pleural effusion. Ultrasound Obstet Gynecol 2010;36:58-64.
13 Knox EM, Kilby MD, Martin WL, Khan KS: In utero pulmonary drainage in the management of primary hydrothorax and congenital cystic lung lesion: a systematic review. Ultrasound Obstet Gynecol 2006;28:726-734.

14 Okawa T, Takano Y, Fujimori K, Yanagida K, Sato A: A new fetal therapy for chylothorax: pleurodesis with OK-432. Ultrasound Obstet Gynecol 2001;18:376-377.

15 Tanemura M, Nishikawa N, Kojima K, Suzuki Y, Suzumori K: A case of successful fetal therapy for congenital by intrapleural infection of OK-432. Ultrasound Obstet Gynecol 2001;18:371-375.

16 Jorgensen C, Brocks V, Bang J, Jorgensen FS, Ronsbro L: Treatment of severe fetal chylothorax associated with pronounced hydrops with intrapleural injection of OK-432. Ultrasound Obstet Gynecol 2003;21:66-69.

-17 Tsukihara A, Tanemura M, Suzuki Y, Sato T, Tanaka T, Suzumori K: Reduction of pleural effusion by OK-432 in a fetus complicated with congenital hydrothorax. Fetal Diagn Ther 2004;19:327-331. 
18 Chen M, Chen CP, Shih JC, Chou HC, Yu CL, Wang BT, Hsieh CY: Antenatal treatment of chylothorax and cystic hygroma with OK-432 in non-immune hydrops fetalis. Fetal Diagn Ther 2005;20:309-315.

19 Chen M, Shih JC, Wang BT, Chen CP, Yu CL: Fetal OK-432 pleurodesis: complete or incomplete. Ultrasound Obstet Gynecol 2005: 26:789-796.

20 Chen M, Hsieh CY, Shih JC, Chou CH, Ma GC, Chen TH, Lee TH, Tsai HD, Cameron AD, Chen CP: Proinflammatory macrophage migratory inhibition factor and interleukin-6 are concentrated in pleural effusion of human fetuses with prenatal chylothorax. Prenat Diagn 2007:27:435-441.

21 Nygaard U, Sundberg K, Nielsen HS, Hertel S, Jorgensen C: New treatment of early fetal chylothorax. Obstet Gynecol 2007;109:1088-1092.

-22 Yang YS, Ma GC, Shih JC, Chen CP, Chou CH, Yeh KT, Kuo SJ, Chen TH, Hwu WL, Lee $\mathrm{TH}$, Chen M: Experimental treatment of bilateral fetal chylothorax using in utero pleurodesis. Ultrasound Obstet Gynecol 2012;39:56-62.
23 Cameraon A, Overton T, Martin W, Kumar S, Fisk N, Walkinshaw S, Taylor M, Robson S: Interventional procedure overview of insertion of pleural-amniotic shunt for fetal pleural effusion. Interventional Procedures Advisory Panel. National Institute for Health and Clinical Excellence (NICE), 2006. http://www. nice.org.uk/.

24 Unger S, Salem S, Wylie L, Shah V: Newborn frontal horn cysts: cause for concern? J Perinatol 2011;31:98-103.

25 Smith M, Zimmerman B, Burke D, Bauman N, Sato Y, Smith R: Efficacy and safety of OK432 immunotherapy of lymphatic malformations. Laryngoscope 2009;119:107-115.

$\longrightarrow 26$ Galinsky R, Polglase GR, Hooper SB, Black MJ, Moss TJM: The consequences of chorioamnionitis: preterm birth and effects on development. J Pregnancy 2013;2013:412831.

27 Olsnes C, Stavang H, Brokstad K, Olofsson J, Aarstad HJ: Chemokines are secreted by monocytes following OK-432 (lyophilized Streptococcus pyogenes) stimulation. BMC Immunol 2009;10:6.

28 Ryoma Y, Moriya Y, Okamoto M, Kanaya I, Saito M, Sato M: Biological effect of OK-432 (Picibanil) and possible application to dendritic cell therapy. Anticancer Res 2004;24: 3295-3302.

29 Ogita K, Taguchi T, Suita S: Experimental study concerning safety dosage of OK-432 for intrauterine treatment. Asian J Surg 2006;29: 202-206.
30 Cowie RV, Stone PR, Parry E, Jensen EC, Gunn AJ, Bennet L: Acute behavioural effects of intrapleural OK-432 (Picibanil) administration in preterm fetal sheep. Fetal Diagn Ther 2009;25:304-313.

-31 Bennet L, Cowie RV, Stone PR, Barrett R, Naylor AS, Blood AB, Gunn AJ: The neural and vascular effects of killed Su-Streptococcus pyogenes (OK-432) in preterm fetal sheep. Am J Physiol Regul Integr Comp Physiol 2010;299:664-672.

32 Stevenson DA, Pysher TJ, Ward RM, Carey JC: Familial congenital non-immune hydrops, chylothorax, and pulmonary lymphangiectasia. Am J Med Genet A 2006;140: 368-372.

33 Ma GC, Liu CS, Chang SP, Yeh KT, Ke YY, Chen TH, Wang B, Kuo SJ, Shih JC, Chen M: A recurrent ITGA9 missense mutation in human foetuses with severe chylothorax: possible correlation with poor response to fetal therapy. Prenat Diagn 2008;28:1057-1063.

34 Huang XZ, Wu JF, Ferrando R, Lee JH, Wang YL, Farese RV Jr, Sheppard D: Fatal bilateral chylothorax in mice lacking the integrin $\alpha_{9} \beta_{1}$. Mol Cell Biol 2000;20:5208-5215. 